Journal of Computer Science 1 (1): 63-71, 2005

ISSN 1549-3636

(C) Science Publications, 2005

\title{
Design and Analysis of a Smart Multi Purpose Electronic Nose System
}

\author{
${ }^{1}$ Mahmoud Z. Iskandarani and ${ }^{2}$ Nidal F. Shilbayeh \\ ${ }^{1}$ Faculty of Science and Information Technology, Al-Zaytoonah Private University of Jordan \\ P.O. Box 911597, Post Code 11191, Jordan \\ ${ }^{2}$ Faculty of Computer Science and Information Technology, Applied Science University \\ P.O. Box 41, Post Code 11931, Amman, Jordan
}

\begin{abstract}
An electronic nose system for multi application purposes is designed and tested. Our hardware design allows various types of sensors to be used for different applications. The system is capable of being interfaced to both analog and digital instruments with special filtering devices that isolate the system from surrounding signals. The design of the system is distinguished through the use of two logic controlled micro fans that stabilize the system atmosphere, which surrounds the smelling sensor and also serves an important function of removing any adsorbed odors on the surface of the sensor. Comprehensive mathematics are used to design and describe each part of the presented system, which allows methodical development and changes of the system parameters for future advances. Testing of the hardware was carried out under computer control using various TGS sensors such as TGS822, and TGS824 and TGS30. Database based software with neural network facility was designed to interface the built hardware and to process the electronic nose signals before being classified.
\end{abstract}

Key words: Electronic Nose, Olfactory, Gas Sensor, Hardware, Software, Neural, Back-Propagation

\section{INTRODUCTION}

The main motivation for electronic noses is the development of qualitative, low-cost, real-time, and portable methods to perform reliable, objective, and reproducible measures of volatile compounds and odors. In order to develop an electronic nose, it is useful to examine the physiology behind olfaction since biological olfactory systems contain many of the desired properties for electronic noses. Also, the contrast between an artificial system and physiology is necessary to achieve a reliable, subjective, and analytically acceptable system [1].

The potential uses of nose-machines, which essentially mimic the functions of human noses but with more precision, are endless. Perfume makers are already using them to protect their patented smells against fakefragrance merchants, and US dockside inspectors have used a high-tech snout to resolve disputes with fishermen over the grading of their catch.

More exciting are the possible medical applications. Scientists are researching the use of electronic noses to diagnose illness by smelling patients' breath with the possibility of installing tiny electronic noses in phone receivers, so that patients can simply breathe into the phone and wait for a diagnosis. A similar smelltransmission device may soon allow surfers on the Internet to "wake up and smell the coffee quite literally. Researchers are investigating the use of breath analysis to identify the stages of the female menstrual cycle: the ability of electronic noses to detect ovulation could benefit both fertility treatment and birth control. High- tech sniffers may be used not just for breath smelling but also to detect other subtle changes in body odor that can indicate disease conditions.

Our unique personal body-odor may also become an alternative form of identification, signaling the end of credit-card fraud, forgotten or misappropriated PIN numbers, fake ID cards, etc. The Association for Payment Clearing Services, an organization set up to find solutions to these problems, is investigating the use of electronic noses in banks, and companies may soon be able to replace security entry systems involving cards and codes with a device that recognizes each employee's personal odor [2].

So far, the electronic noses available are no more sensitive than the average human nose - although specialist noses are being developed - but electronic noses do have significant advantages over those attached to humans. Electronic noses do not get bored with repetitive smelling tasks, or de-sensitized through habituation to particular odors. Unpleasant smells such as industrial chemicals and sewage do not make electronic sniffers feel sick, and their performance on smelling tasks does not fluctuate according to mood, hormone cycles or other unpredictable human factors [3].

In this study, a fully operational electronic nose system for general smelling applications, which models the function of the biological nose, is presented.

Background: The mammalian olfactory system uses a variety of chemical sensors, known as olfactory receptors, combined with automated pattern recognition 
incorporated in the olfactory bulb and olfactory cortex in the brain [4-5]. No one-receptor type alone identifies a specific odor. It is the collective set of receptors combined with pattern recognition that results in the detection and identification of each odor. Fig. 1 illustrates the major components and function of the mammalian olfactory system and its sensory components. Odor molecules arrive at the olfactory receptors stimulating an electro-chemical response that is transmitted through the crib form plate to the olfactory bulb and ultimately the olfactory cortex.

The major operations olfaction can be broken into sniffing, reception, detection, recognition, and cleansing of odors. The olfaction process begins with sniffing, which brings odorant molecules from the outside world into the nose. With the aid of turbinated (bony structures in the nose which produce turbulence), sniffing also mixes the odorant molecules into a uniform concentration and delivers these molecules to the mucus layer lining the olfactory epithelium in the upper portion of the nasal cavity. Next, the odorant molecules dissolve in this thin mucus layer which then transports them to the cilia (hair like fibers) of the olfactory receptor neurons. The mucus layer also functions as a filter to remove larger particles.

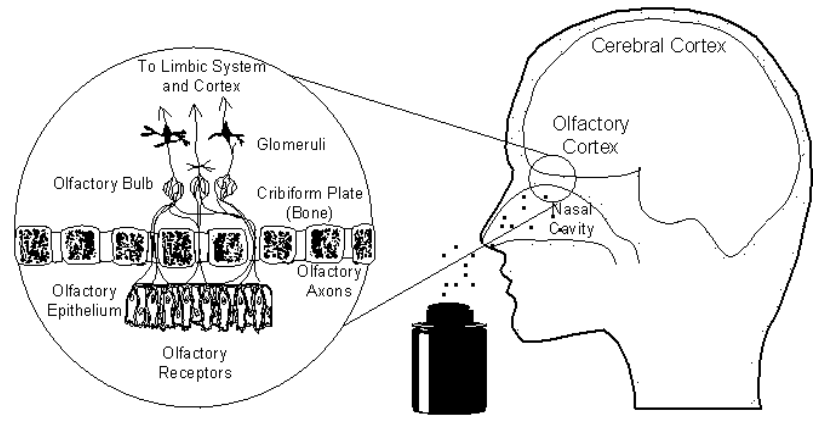

Fig. 1: Major Components of the Senses of Olfaction in the Human

Fig. 1 also illustrates the major components of the senses of olfaction and taste in the human. The major olfactory components are the olfactory receptors (sensors), the olfactory bulb (signal pre-processing), and the olfactory cortex (odor identification). The VNO is the vomero nasal organ and is associated with pheromone detection.

Reception involves binding the odorant molecules to the olfactory receptors. These olfactory receptors respond chemically with the odorant molecules. This process involves temporarily binding the odorant molecules to proteins that transport the molecules across the receptor membrane. Once across the boundary, the odorant molecules chemically stimulate the receptors. Receptors with different binding proteins are arranged randomly throughout the olfactory epithelium.

The chemical reaction in the receptors produces an electrical stimulus. These electrical signals from the receptor neurons are then transported by the olfactory axons through the crib form plate (a perforated bone that separates the cranial cavity from the nasal cavity within the skull) to the olfactory bulb (a structure in the brain located just above the nasal cavity). From the olfactory bulb, the receptor response information is transmitted to the olfactory cortex where odor recognition takes place. After this, the information is transmitted to the limbic system and cerebral cortex. There are no individual olfactory receptors or portions of the brain that recognize specific odors. It is the brain that associates the collection of olfactory signals with the odor.

Finally, in order for the nose to respond to new odors, the olfactory receptors must be cleansed. This involves breathing fresh air and the removal of odorant molecules from the olfactory receptors.

The Smart Electronic Nose System (SENS): The two main components of the SENS are the sensing system and the automated pattern recognition system as shown in Fig. 2. This combination of broadly tuned sensors coupled with sophisticated information processing makes the electronic nose a powerful instrument for odor analysis applications. The sensing system can be an array of chemical sensors where each sensor measures a different property of the sensed chemical, or it can be a single sensing device (e.g., gas chromatograph, spectrometer) that produces an array of measurements for each chemical, or it can be a hybrid of both. Each odorant or volatile compound presented to the sensor array produces a signature or characteristic pattern of the odorant [5-7].

By presenting many different odorants to the sensor array, a database of signatures is built up. This database of odorant signatures is then used to build the odor recognition system. The goal of this process is to train or configure the recognition system to produce unique classifications or clustering's of each odorant so that an automated identification can be implemented. Like biological systems, electronic noses are qualitative in nature and do not give precise concentrations. Unlike biological systems, current electronic noses are usually trained to identify only a few different odors or volatile compounds. Also, current systems lack the temporal dynamics found in biological systems and neuromorphic models.

During operation, a chemical vapor or odor is blown over the sensor array, the sensor signals are digitized and fed into the computer, and the Artificial Neural Networks [3] (implemented in software) then identifies the chemical as shown in Fig. 3. 


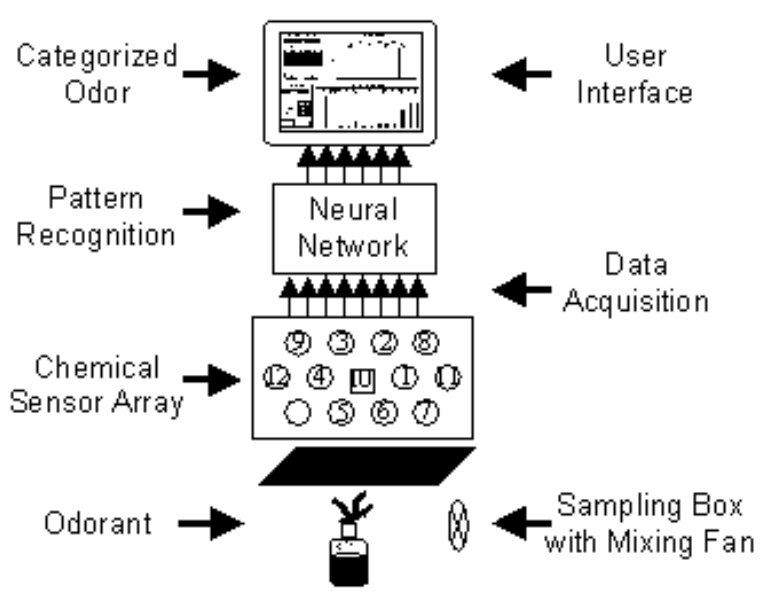

Fig. 2: Schematic of SENS

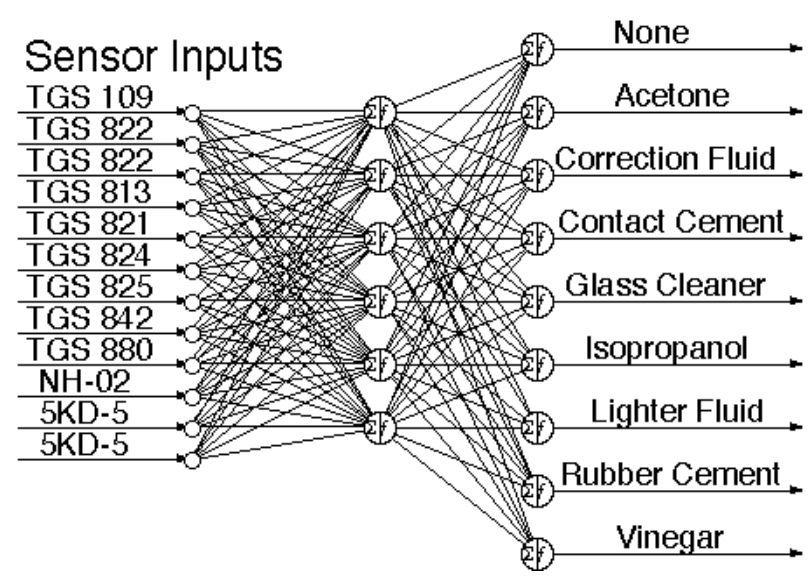

Fig. 3: Neural Networks Recognition Engine

System Hardware Design: The Smart Electronic Nose system (SENS) is a system that converts the sensed odor in air to an electrical signal that is conditioned and sent to a computer to be interpreted and classified using a specifically designed Neural Network algorithms. Fig. 4 shows the deigned and built SENS circuit, which consists of the following parts:

Sensing Unit: The used sensing element is a Figaro gas sensor, which consists of a Tin Oxide $\left(\mathrm{SnO}_{2}\right)$ semiconductor [8], which has low conductivity in clean air. In the presence of a detectable gas, the sensor's conductivity increases depending on the gas concentration in the air. A simple electrical circuit can convert the change in conductivity to an output signal, which corresponds to the gas concentration. The used TGS830 has high sensitivity to the vapors of organic solvents as well as other volatile vapors. It also has sensitivity to a variety of combustible gases such as carbon monoxide, making it a good general-purpose sensor. The sensor is also manufactured with a ceramic base which is highly resistant to severe environments. The sensing unit is shown in Fig. 5.

The relationship between sensor resistance and the concentration of detected odor can be expressed by the following equation over a predetermined range of odor concentrations.

$\mathrm{R}=\mathrm{A}[\mathrm{C}]-\mathrm{B}$

Where:

$\mathrm{R}=$ electrical resistance of the sensor

$\mathrm{A}, \mathrm{B}=$ constant

$[\mathrm{C}]=$ Odor concentration

The input to the odor sensor(s) can either be AC or DC of maximum value of $24 \mathrm{~V}$ maximum power is $15 \mathrm{~mW}$. The maximum input to the heater is $5 \mathrm{~V}$. The outputs of the sensor(s) are:

* Using DC input: only change of the voltage amplitude of the output with change gas.

* Using AC input: the change will be in the amplitude and phase.

The sensor resistance (RS) is calculated using the following formula:

$$
R_{S}=\left(\frac{V_{C}}{V_{R L}}-1\right) \mathrm{R}_{\mathrm{L}}
$$

With Power dissipation across sensor electrode (PS) given by:

$$
P_{S}=\mathrm{V}_{C}^{2} * \frac{R_{S}}{\left(R_{S}+R_{L}\right)^{2}}
$$

In this study an $\mathrm{AC}$ source with $10 \mathrm{KHz}$ frequency is used.

Function Generator Unit: Fig. 6 shows the function generator used to input signal to the sensing unit where it gets modulated the smelled odor. The Unit employs the XR-2206 monolithic function generator (Fig. 7), which is capable of producing accurate sinusoidal, square, triangular, and ramp waveforms. This makes this generator ideal for communication and instrumentation purposes specially for our electronic nose applications that may require different types of signals that can also be pre-modulated before its inputted to the sensor using analogue or digital modulation techniques. The output waveforms of the XR-2206 can be both amplitude of frequency 




Fig.4: The SENS Circuit 



Fig. 5: Smell Sensor and Equivalent Electrical Circuit

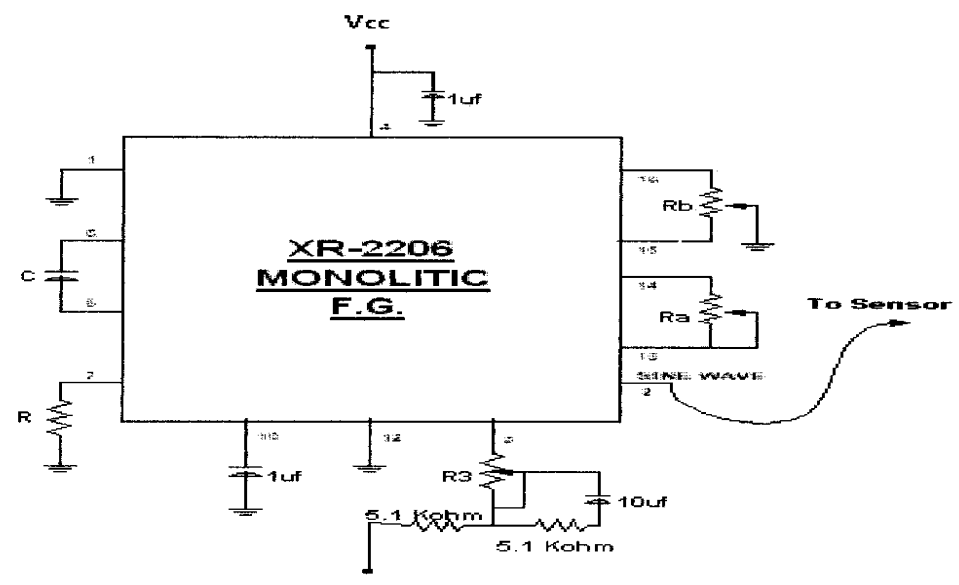

Fig. 6: The Function Generator Unit

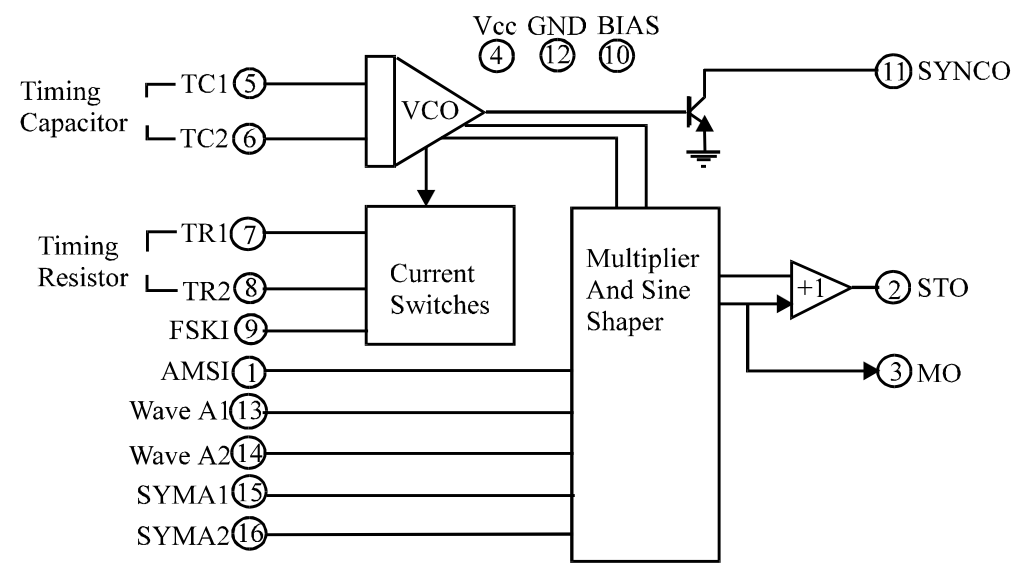

Fig. 7: XR-2206 Function Generator

modulated by an external signal. The operating frequency can be selected over the range $.01 \mathrm{~Hz}$ to 1 $\mathrm{MHz}$.

When used in our system Fig. 6, $\mathrm{R}_{1}$ at pin 7 provides the desired frequency tuning. The harmonic content of the used sinusoidal output can be reduced by additional adjustment as shown in Fig. $6 . R_{a}$ adjusts the shape of the sine wave with $R_{b}$ providing the required adjustment to maintain waveform symmetry.

The frequency of operation $f_{o}$ is determined by an 
external timing capacitor $\mathrm{C}$ across pins 5 and 6 and by a resister $\mathrm{R}$ connected to either pin 7 or 8 . The operating frequency is given by:

$$
f_{o}=\frac{1}{R * C} H z
$$

And can be adjusted by varying either R or C. Stability is optimum for $4 \mathrm{~K}<\mathrm{R}<200 \mathrm{~K}$ with values of $\mathrm{C}$ in the range of $1000 \mathrm{pF}<\mathrm{C}<100 \mathrm{uF}$. Our sinusoidal carrier frequency of $10 \mathrm{KHz}$ is chosen to derive the system with $\mathrm{R}=4.34 \mathrm{~K}$ and $\mathrm{C}=22 \mathrm{uF}$ which when substituted in equation 4 gives an operating frequency of $10.473 \mathrm{KHz}$. the maximum amplitude of the output signal of the generator is inversely proportional to the resistor $R_{3}$ (Fig. 6) connected to pin 3. Hence, by using $\mathrm{R}_{3}=50 \mathrm{~K}$ would obtain an approximate value of 6 volts for our generated sine wave.

The Filtering Unit: Fig. 8 shows the multistage BPF used to noise remove and frequency specify the sensing unit output signal. This means that any signal outside the prescribed bandwidth will not be accepted. This is essential for medical and security signatures. This circuit, which is based on the concept of two integrators, cascaded together with an overall feedback loop (two integrator loop bi-quad) possesses high selectivity necessary for our applications.

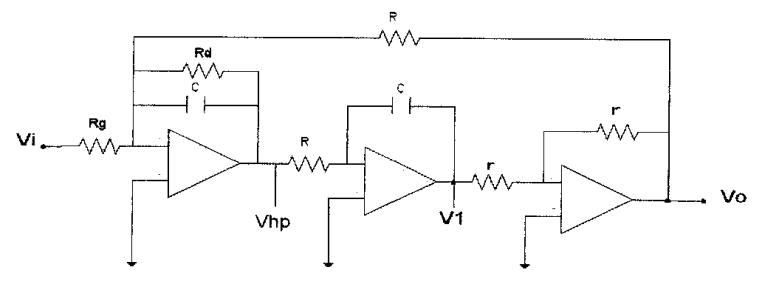

Fig. 8: BPF System

To mathematically describe the filtering circuit we consider first the second order high pass transfer function given by:

$$
\frac{V_{h p}}{V_{i}}=\frac{K S^{2}}{S^{2}+\left(\frac{\omega_{0}}{Q}\right) S+\omega_{0}^{2}}
$$

Where:

$\mathrm{K}$ is a gain constant.

Q: is a quality factor.

Cross multiplying equation (5) gives

$V_{h p} S^{2}+\left(\frac{\omega_{0} v_{h p}}{Q}\right) S+V_{h p} \omega_{0}^{2}=V_{i} K S^{2}$
Divide both side of equation (6) by $\mathrm{S}^{2}$ gives:

$V_{h p}+\frac{1}{\mathrm{Q}}\left(\frac{\omega_{0}}{S} V_{h p}\right)+\left(\frac{\omega_{0}^{2}}{S^{2}} V_{h p}\right)=\mathrm{KV}$

In equation (7) we observe that the signal $\frac{\omega_{0}}{S} V_{h p}$ can be obtained by passing $V_{h p}$ through an intetegrator with a time constant equal to $\frac{1}{\omega_{0}}$.

Furthermore, passing the resulting signal through another identical integrator results $\frac{\omega_{0}^{2}}{S^{2}} V_{h p}$. Fig. 9 shows the block diagram from such a two integrator arrangement.

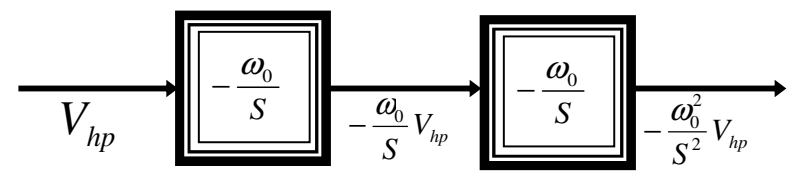

Fig. 9: Two Integrator Arrangement

In order to obtain $\mathrm{V}_{\mathrm{hp}}$ (the input signal feeding the two cascaded integrators), equation (7) is rearranged which gives:

$V_{h p}=\mathrm{KV}_{\mathrm{i}}-\frac{1}{\mathrm{Q}}\left(\frac{\omega_{0}}{S} V_{h p}\right)-\left(\frac{\omega_{0}^{2}}{S^{2}} V_{h p}\right)$

Which suggests that $\mathrm{V}_{\mathrm{hp}}$ can be obtained by using the weighted summer as shown in Fig. 10.

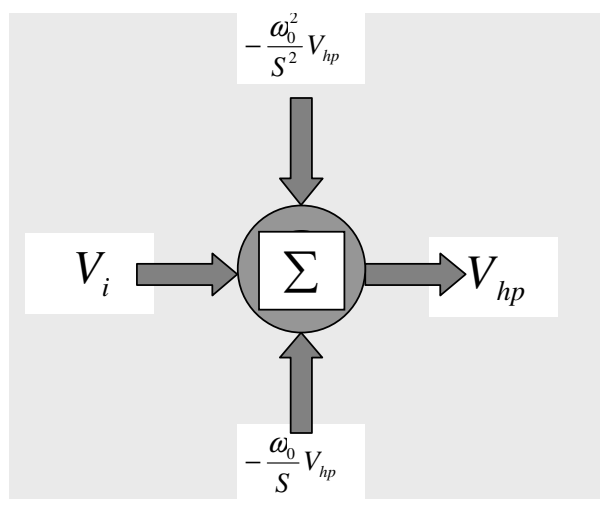

Fig.10: The Weighted Summer

Considering Fig. 9 and 10 we obtain Fig. 11 which is described by the following expression: 


$$
V_{h p}=\frac{\left(\frac{-\omega_{0}}{S}\right) V_{h p}}{V_{i}}=\frac{K \omega_{0} S^{2}}{S^{2}+\left(\frac{\omega_{0}}{Q}\right) S+\omega_{0}^{2}}
$$

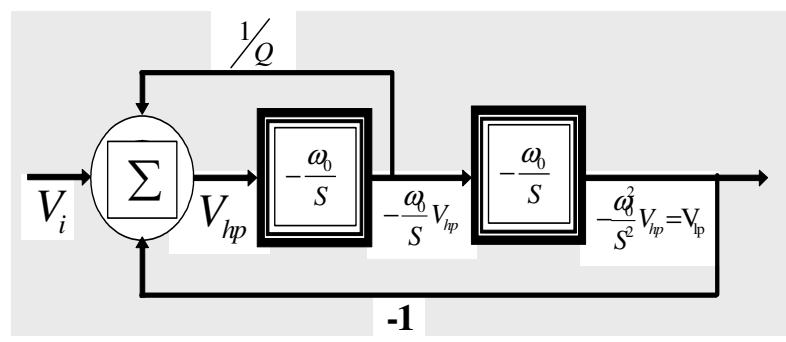

Fig.11: Two Integrator Biquad

Equation 9 indicates that the output signal of the first integrator is $V_{b p}$ with a center frequency gain of the realized band pass filter equals to $-\mathrm{KQ}$.

Amplification Unit: After the signal passes through the bi-quad BPF it needs simple amplification process to enhance the voltage level of the modulated sinusoidal signal coming out of the sensor. Fig. 12 shows the OpAmp used enlarge the sensing unit filtered signal.

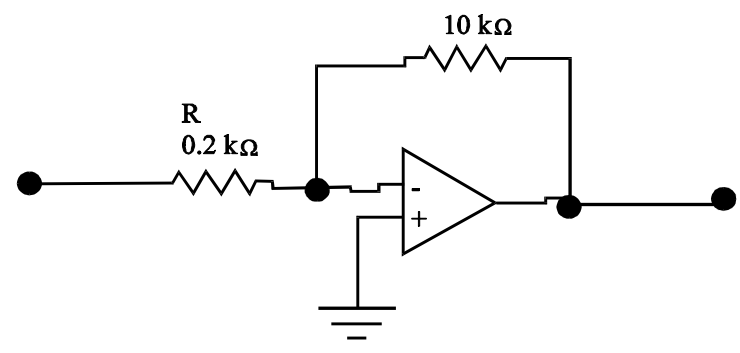

Fig. 12: Amplification Unit

The output voltage of the circuit described in Fig. 12 is given by:

$$
V_{0}=-\frac{R_{f}}{R} V_{1}
$$

Where, $\mathrm{R}_{\mathrm{f}}=10 \mathrm{~K}$ and $\mathrm{R}=0.2 \mathrm{~K}$.

This substituting the above values will give $\mathrm{K}=50$. This value of $K$ is used together with a $Q$ value of 20 to obtain the required center frequency gain of the band pass filter.

Analogue to Digital Converter Unit: This unit is used to enable the sampling and processing of the electronic nose signal by the specifically designed software and neural engine. It interfaces the system hardware to its interpreting and classifying software. This is carried out using the ADC0804 device shown in Fig. 13. To select the required sampling frequency, external components are needed. Fig. 14 shows the necessary connections for our system ADC.

The design of analog to digital converter unit (Fig. 14) is based on frequency sampling with voltage reference as carried out by the ADC 0804 (Fig. 13). For our application a $640 \mathrm{KHz}$ frequency is selected with an external clock supplied to the ADC unit by our computing system CPU.

As shown in Fig. 14 an external capacitor and resister are required for circuit operation. Using the expression $F_{c l k}=\frac{1}{R_{1} C_{1}}$ and knowing the value of the capacitor $150 \mathrm{pF}$ will give us a resistance value of $10 \mathrm{~K}$. A reference voltage of 2.5 volts is used.

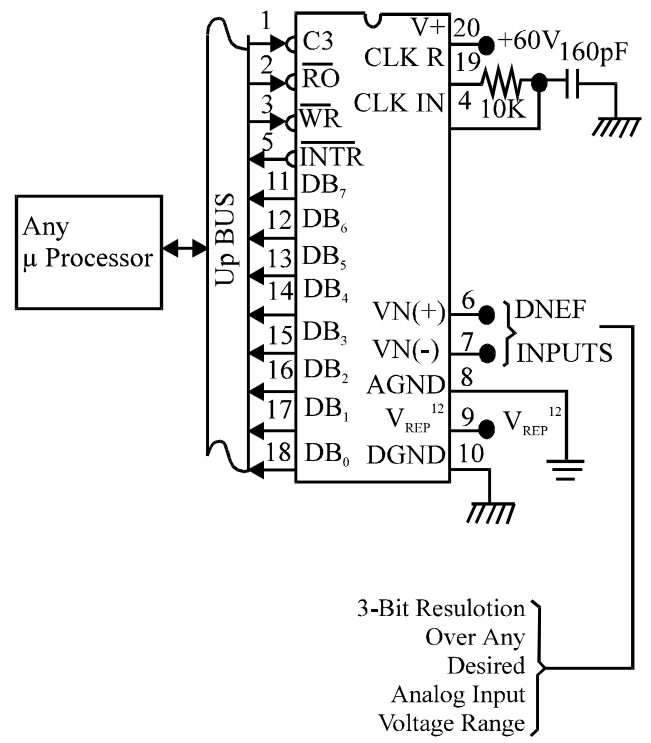

Fig. 13: ADC0804

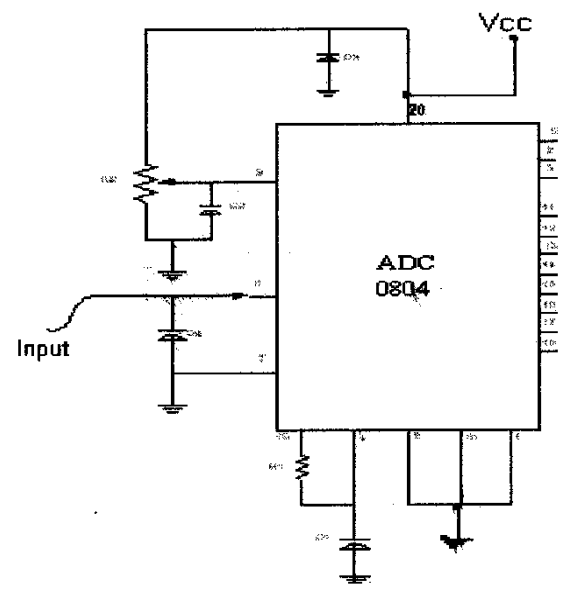

Fig. 14: ADC0804 Unit 
To help the sensing unit recover to its original status after smelling odors, and to eliminate problems connected to mixtures of odors and misclassification, a computer controlled ventilation and extraction system is implemented, whereby two micro fans are used to stabilize the air over the sensing unit and extract any remained molecules on the surface of the sensor element. Following are the main parts of the extraction and air stabilization system.

Timing Unit: Fig. 15 shows the used 555 Timer, which is used to start and stop the micro fans under computer control. The 555 timer needs external components to set its switching time characteristics as shown in Fig.16.

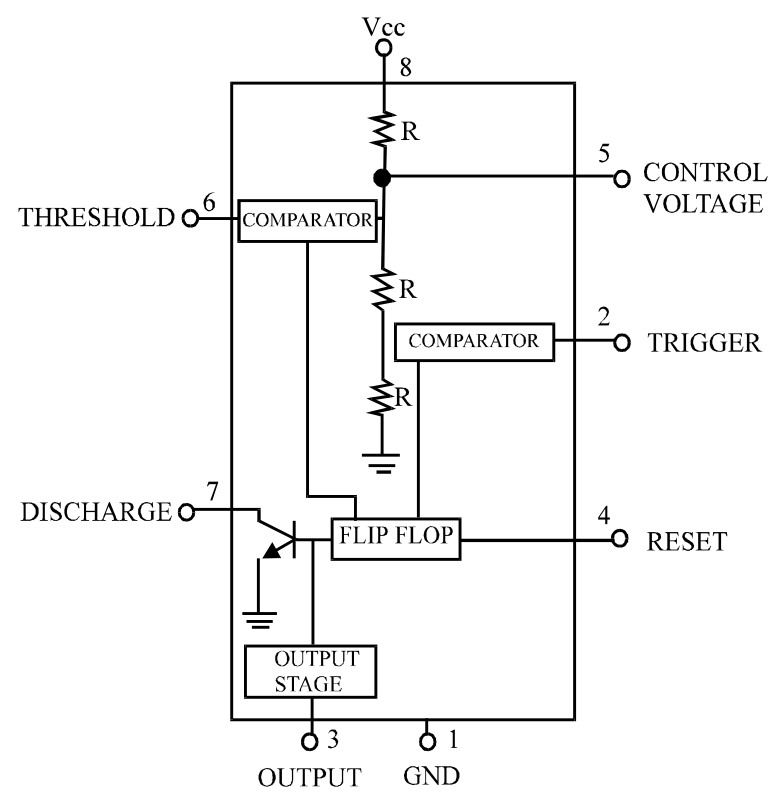

Fig. 15: The Used 555 Timer

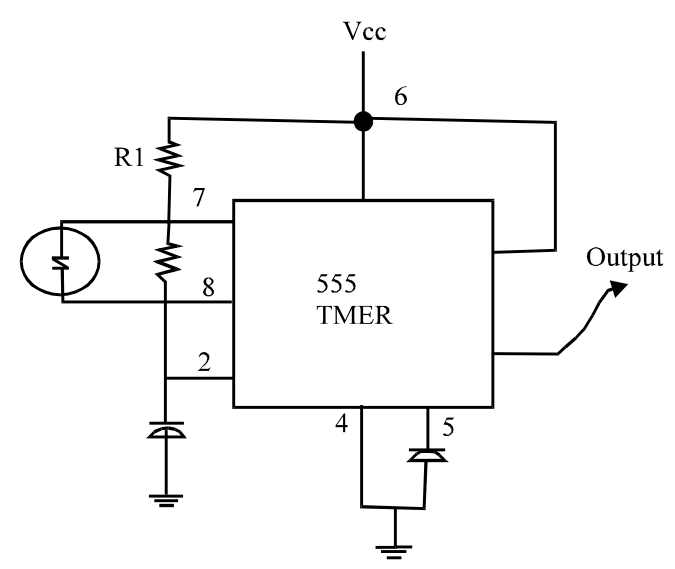

Fig. 16: The 555 Timing Unit
The output pulse frequency of the timing unit shown in Fig. 16 and generated by 555 timer in Fig. 15 is controlled by $R_{1}, R_{2}$, and $C_{1}$ the period of the pulse can be adjusted using $R_{1}$ and $C_{1}$ using the following expression $\mathrm{T}_{\mathrm{ch}}=\mathrm{R}_{1} * \mathrm{C}_{1} * \ln 2$. This function is used to cover the rising part of the waveform with the disgorging part given by $\mathrm{T}_{\mathrm{dis}}=\mathrm{R}_{2} * \mathrm{C}_{1} \ln 2$.

Counting and Logic Control Unit: Fig. 17 shows the counting circuit, which is necessary to produce the following codes to control the internal process of the designed hardware. The unit will produce codes from 00 to 101 . These codes are programmed as shown in table.1. Fig. 18 shows the implementation of counter and logic in our system.

The 7493IC consists of 4 flip-flops. The flip-flops can be connected to count in binary, PCD or other counting formats. It can be connected to count from 0 to different maximum values. Yhis is done by connecting 1 or 2 of the outputs to reset inputs at $R_{1}$ and $R_{2}$ (pins 2 and 3). Fig.18 shows QA (pin12) and QB (pin 9) having a NAND gate input and an output which connects with $\mathrm{R}_{1}$ and $\mathrm{R}_{2}$. the output from the timing unit is connected to the input of the counter (pin 14). The overall circuit shown in Fig. 18 is used to derive the micro fans with a driving current obtained through the circuit in Fig. 19 and described by the following equation:

$$
I_{0}=\left(1+\frac{R_{1}}{R_{2}}\right) I_{i}
$$

Table 1: Control Codes of Designed Hardware

\begin{tabular}{ll}
\hline Code & Control Function \\
\hline 00 & Operate Fan Number 1 \\
01 & Reset Interval \\
10 & ADC Conversion \\
11 & Operate Fan Number 2 \\
100 & Not used \\
101 & Reset \\
\hline
\end{tabular}

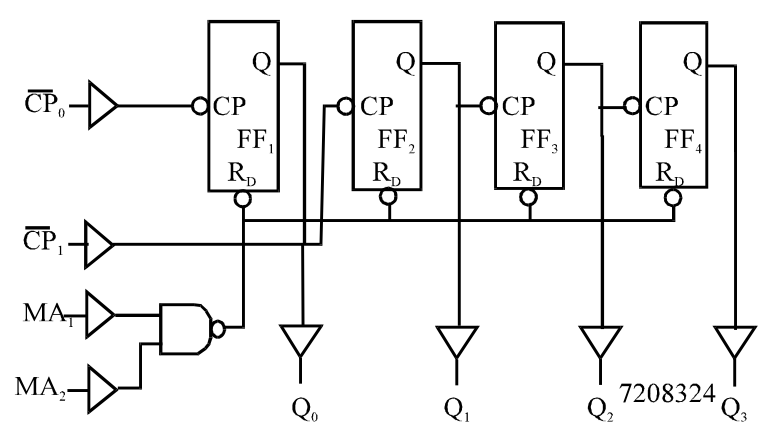

Fig.17: 7493 Counting Circuit 


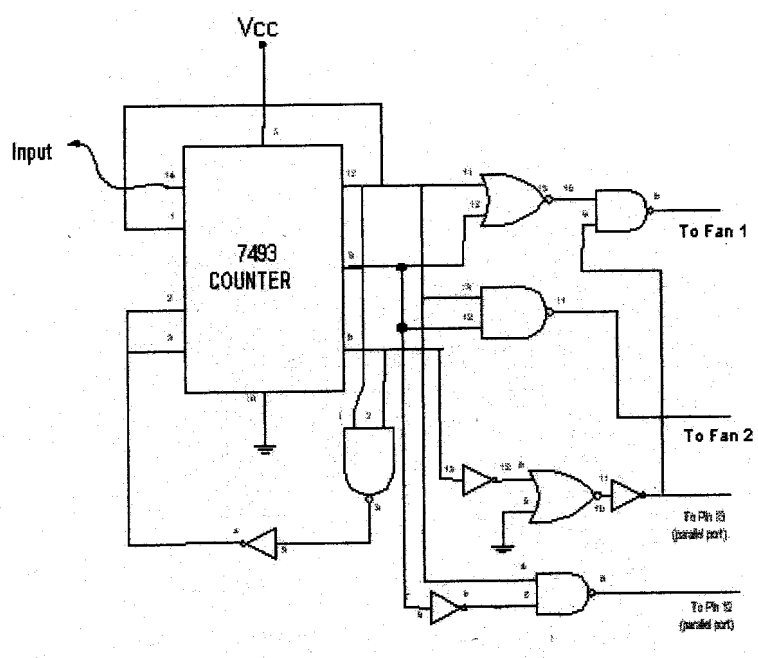

Fig.18 Counting and Logic Control Unit

Fig. 19 shows the driving units for the micro fans. This circuit is necessary to pull the voltage and current levels to their right values for the fans to be driven correctly.



Fig.19: Fans Driving circuit

\section{CONCLUSION}

The designed and built TGS 800 series Smart Electronic Nose System (SENS) proved to be an excellent system for the general purpose applications as it allows any of the 800 series sensors to be interfaced without the need for any hardware modification or adjustment. The extensive use of mathematical equations describing each step of the design is a major advantage in our designed system as it allows easy modification and development of the system to incorporate any newly presented ideas and components on the market. The initial choice of the TGS type of sensors is due to there simple design and the advantage of having an integrated heater which helps in stabilizing detecting element temperature and evaporation of adsorbed odors molecules, hence provides acceptable results that is improved through the use of hardware filtering and digitizing devices and an intelligent software which provides excellent classification. The overall designed system performance is further improved through the use of our specially controlled micro fans that enable rapid recovery of detected signals to its initial levels. Further improvement could be introduced to our efficient system by integrating all sensors onto one device where by integration and miniaturization will improve the electrical characteristics of the sensing part of the designed system.

\section{REFERENCES}

1. Nagle, H. T., 1998. The How and Why of Electronic Noses. IEEE Spectrum, pp: 22-33.

2. Staples, E. J., 1998. Dioxin/Furan Detection and Analysis Using a SAW-Based Electronic Nose. Proc. IEEE International Ultrasonic Symposium, Sendai, Japan.

3. Keller, P., 1994. Three Neural Network Based Sensor Systems for Environmental Monitoring. Proc. IEEE Electro94 Conference, Boston.

4. Merler, S., C. Furlanello, B. Larcher and A. Sboner, 2001. Tuning Cost Sensitive Boosting and its Application to Melanoma Diagnosis. In MCS 2001, Cambridge, UK, Vol 2096 of LNCS, pp: 32-42.

5. Pardo, M. and G. Niederjaunfner, 2000. Data Preprocessing Enhances the Classification of Different Brands of Espresso Coffee with an Electronic Nose. Sensors \&Actuators B, 69.

6. Deveza, R., D. Thiel, R.A. Russel and A. Mackay-Sim, 1994. Odor Sensing for robot guidance. The Int. J. Robotics Res., 13: 232-239.

7. Shilbayeh, N. and M.Iskandarani, 2003. Discrimination of Perfumes Using an Electronic Nose System. WSEAS Trans. On Circuits and Systems, 2: 820-825.

8. Figaro sensors (www.figaro.com). 